\title{
Prevalence, correlates and course of behavioural and psychological symptoms of dementia in the population ${ }^{\dagger}$
}

George M. Savva, ${ }^{*}$ Julia Zaccai, ${ }^{*}$ Fiona E. Matthews, Julie E. Davidson, Ian McKeith, Carol Brayne, of behalf of the Medical Research Council Cognitive Function and Ageing Study

\section{Background}

Behavioural and psychological symptoms of dementia (BPSD) are major contributors to the burden of dementia.

\author{
Aims \\ To describe the prevalence, correlates and course of BPSD in \\ the population of England and Wales.
}

\section{Method}

The prevalence of 12 symptoms was estimated in 587 participants with dementia and 2050 participants without dementia as part of a population-based longitudinal study of ageing. The effect of risk factors and the factor structure were estimated using 1782 interviews provided by participants with dementia throughout the study.

\section{Results}

Each symptom apart from sleeping problems was more common in the population with dementia. The co-occurrence of the symptoms was explained by a four-factor solution, corresponding to psychosis/apathy, depression/anxiety, irritability/persecution and wandering/sleep problems. Psychosis occurred more frequently with declining cognition. Anxiety and depression were more common in younger individuals and in those with poor self-reported health. Persistence varied between symptoms.

\section{Conclusions}

Behavioural and psychological symptoms of dementia affect nearly all people with dementia. Symptoms co-occur, and the symptoms that affected individuals experience are related to their socio-demographic and clinical characteristics.

\section{Declaration of interest}

This study was funded by a grant from GlaxoSmithKline. The Cognitive Function and Ageing Study is supported by the Medical Research Council.
Behavioural and psychological problems affect almost all people with dementia at some point during the progression of the disorder. ${ }^{1}$ They result in long-term hospitalisation, increased medication and decreased quality of life for caregivers and patients. ${ }^{2}$ It is not clear whether behavioural and psychological symptoms of dementia (BPSD) are epiphenomena of the neurodegenerative process or are the symptoms of specific, biologically distinct subtypes of dementing disorders. Understanding the prevalence and risk factors for BPSD is a first step in developing treatment regimes and policies for their management. Few studies of BPSD prevalence have been conducted in a population setting, ${ }^{3-5}$ and inconsistent assessment and ascertainment methods and the use of different sources for the study cohorts have led to estimates of the prevalence of BPSD that differ enormously. Cross-sectional studies have shown that different symptoms are more common at different stages of dementia ${ }^{6-9}$ and suggest a complex course of BPSD that must be examined in a longitudinal setting.

The Medical Research Council Cognitive Function and Ageing Study (MRC CFAS) offers a setting that overcomes many of the methodological problems associated with investigating the epidemiology of BPSD. It is a large, prospective, population-based study of health in elderly people using an array of validated questionnaires. In this paper we describe the epidemiology of BPSD in the elderly population of England and Wales. We estimate their prevalence in the 587 study participants diagnosed with dementia at baseline compared with similar symptoms in 2050 participants without dementia. We describe their co-occurrence pair-wise and analyse the pattern of the co-occurrence using factor

†See editorial, pp. 199-200, this issue.

*These authors contributed equally to the work. analysis, and investigate demographic and clinical correlates for each symptom we describe. We further report the profile of BPSD in the 244 participants with dementia at baseline who were available for reassessment 2 years after the baseline interview and the 348 participants who developed dementia within the period between the baseline and follow-up interviews.

\section{Method}

\section{Study population and data sources}

The MRC CFAS has been fully described elsewhere. ${ }^{10}$ It is a multicentre longitudinal population-based study of ageing, with a focus on cognitive and physical decline. Random samples of people aged 65 years and over were contacted at each of five centres representative of rural and urban areas in England and Wales (Cambridgeshire, Gwynedd, Newcastle upon Tyne, Nottingham and Oxford) with an $82 \%$ response rate. A total of 13004 participants were followed.

Figure 1 shows a flow diagram of the interviews in the MRC CFAS from which BPSD could be assessed. Each participant underwent an initial screening interview. A 20\% subsample of those screened, stratified by cognitive ability, was selected for detailed assessment. After 2 years, a re-screen of the entire remaining study population was undertaken and a further $20 \%$ subsample of those not previously assessed underwent the assessment, together with a second assessment interview for those who had previously been assessed. Both assessment groups were followed up again 6 years after the first interview, a subsample of these were reassessed at 8 years, and a full assessment of the entire remaining cohort was undertaken after 10 years. 


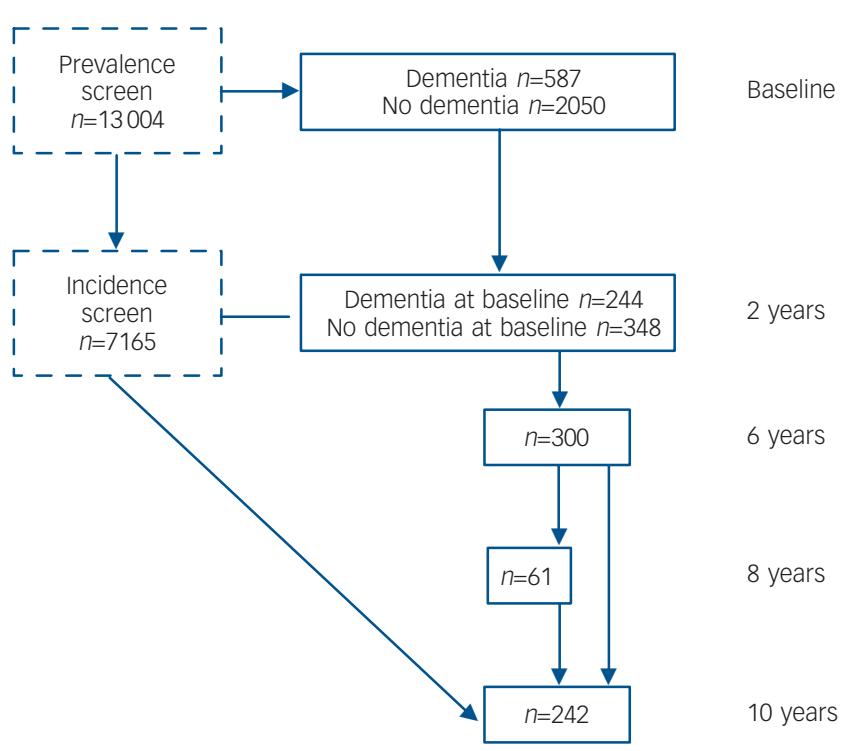

Fig. 1 Flow diagram of the assessment of behavioural and psychological symptoms of dementia (BPSD) in the Medical Research Council Cognitive Function and Ageing Study (MRC CFAS).

Prevalence of BPSD in the populations with and without dementia was estimated using the baseline assessment. The course of BPSD was measured in the 244 participants with dementia at wave 2 who had dementia at baseline and the 348 participants with dementia at wave 2 who had dementia at baseline and the 348 with incident dementia. All available assessments of people with dementia were included in the estimation of risk factors and co-occurrence, with a random effects model used to account for individual participants contributing more than one interview.

Socio-demographic information was collected on all participants at each interview. The screening interview included the Mini-Mental State Examination (MMSE) ${ }^{11}$ and Automated Geriatric Examination for Computer-Assisted Taxonomy (AGECAT) organicity items, ${ }^{12}$ both of which are sensitive to dementia. The detailed assessment included the Geriatric Mental State (GMS) ${ }^{13}$ interview, which was adapted to map the AGECAT and Cambridge Mental Disorders of the Elderly Examination (CAMDEX) ${ }^{14}$ algorithms, and MRC Clinical Information. The History and Aetiology Schedule, which accompanies the GMS, was conducted with caregivers.

\section{Defining dementia and BPSD}

Trained nurses administered the adapted GMS, which comprised 157 items including interviewer observations. These responses were used to generate a study diagnosis of dementia using the AGECAT algorithm. Dementia was defined as an AGECAT organicity rating case level of $\mathrm{O} 3$ or above, which has previously been found to be equivalent to diagnosis by DSM-III-R. ${ }^{12}$

We assessed BPSD using interviews conducted with both study participants and their carers, combined with observations made by the interviewer during the course of the interviews. Each symptom was recorded as either present or absent, with presence determined using the criteria described in Table 1.

The socio-demographic and clinical risk factors we tested were based on a review of the literature and included age; gender; education; social class; institutionalisation; cognitive impairment measured by MMSE; visual or hearing impairment; history of heart attack, stroke, diabetes, brain injury or emotional problems; self-rated health; smoking; alcohol misuse; family history of vascular disease; family history of psychiatric disorders; current emotional state measured by a recent loss of spouse, close friend or pet; and ApoE genotype. Not all risk factors were known for all participants; where they were not known they were assumed to be missing at random.

\section{Analysis}

The prevalence of each symptom in those people with dementia and without dementia was estimated using the baseline assessment, at which 587 participants received a study diagnosis of dementia and 2050 did not have dementia. Participants were back-weighted in the prevalence estimation to adjust for oversampling in the study population of individuals aged 75 years or older and the subsequent stratified sampling for the assessment interview.

Data collected from all baseline and follow-up assessments where a study diagnosis of dementia had been made were used for the analysis of symptom co-occurrence and the estimation of risk factors. Many participants contributed more than one assessment and a random effects model was used for the correlation of symptom profile within each participant. When estimating risk factors and pair-wise symptom co-occurrence we controlled the false discovery rate at 5\%, meaning that approximately $95 \%$ of our reported significant associations are not because of chance. Using the Benjamini-Hochberg procedure, ${ }^{15}$ the $P$-value threshold to achieve this rate given the distribution of $P$-values in our study of risk factors is 0.0107 , and when estimating symptom co-occurrence the cut-off is 0.033 .

Logistic regression was used to calculate the effect of risk factors. Each analysis was also adjusted for age, gender, MMSE score and whether the interviewee had a hearing or eyesight problem sufficient to affect the interview process. Interactions between possible risk factors were not considered. Each of the risk factors was analysed as a binary variable except for age, which was measured in completed years and included as a linear variable, and MMSE score, which was included in three categories corresponding to scores of $0-17,18-21$, and 22-30. Odds ratios for the low and high MMSE categories are reported with respect to the middle category. The most likely cause of a missing MMSE score is very severe cognitive impairment and so any missing MMSE score was assumed to be low.

Odds ratios adjusted for age, gender, MMSE score and whether the interviewee had a hearing or eyesight problem were used to measure the pair-wise co-occurrence between symptoms. Factor analysis was then conducted to reveal the structure of this co-occurrence. The tetrachoric correlation matrix relating the symptoms was found and this was subjected to the principal factor analysis algorithm. The resulting factor solution was rotated for ease of interpretation using the Varimax rotation. Different factor analysis algorithms led to similar results. Scree plots were used to determine the number of factors needed.

We only observe the symptom profile of participants at their baseline and follow-up assessments, and so their incidence and persistence cannot be directly observed. Markov chain models have been used to estimate the persistence and incidence of BPSD based on repeat interviews, ${ }^{16}$ but this method introduces assumptions regarding the homogeneity of participants and the stability of the prevalence of symptoms during the course of dementia that are unlikely to be satisfied. We therefore report for each symptom the number of participants who exhibit the symptom for the first time at the second assessment, and the number of those with dementia at baseline in whom the symptom was also observed at follow-up. Odds ratios were used to assess the effect of symptom presence at baseline on presence at follow-up. Factor scores were calculated for each participant with dementia 
and correlations between factors were found between baseline and 2-year follow-up to assess the temporal relationships between symptoms.

We found no association between refusal to be interviewed at follow-up and age, gender, cognitive impairment or presence of BPSD in the participants with dementia. The only significant indicator for refusal among those with dementia is institutionalisation, with those participants living at home more likely to refuse (48 out of 286) than those living in institutions (17 out of 238). It is possible that institutionalisation also affects the course of BPSD and a sensitivity analysis was conducted to assess any bias on our results.

All of the statistical analysis was performed using STATA 9.2 on Sun Solaris.

\section{Role of the funding source}

Neither the MRC nor GlaxoSmithKline influenced the design, data collection, analysis or interpretation of our findings.

\section{Results}

\section{Cohort description}

Table 1 presents key characteristics of the MRC CFAS cohort at baseline and at 2-year follow-up.

\section{Prevalence of BPSD}

Table 2 shows the estimated prevalence of each symptom according to dementia status in the population over the age of 65 in England and Wales. The prevalence of each symptom is significantly higher in the population with dementia except for sleep problems, which are very common in both groups, and anxiety, which was around $40 \%$ more common in the population with dementia $(P=0.07)$. A more detailed analysis of responses relating to sleep problems revealed that people with dementia self-report fewer sleeping problems, although their carers report significantly more.

\begin{tabular}{|c|c|c|c|c|c|}
\hline & \multicolumn{2}{|c|}{ Group assessed at baseline ${ }^{a}$} & \multicolumn{2}{|c|}{ Participants with dementia } & \multirow{2}{*}{$\begin{array}{l}\text { Incident dementia } \\
\text { cases at } 2 \text { years }\end{array}$} \\
\hline & No dementia & Dementia & Baseline & 2-year follow-up & \\
\hline$n$ & 2050 & 587 & 244 & 244 & 348 \\
\hline Age, years: mean & 74.2 & 81.9 & 83.1 & 84.7 & 83.2 \\
\hline Female, \% & 58 & 70 & 70 & 70 & 67 \\
\hline Living in institutions, \% & 2 & 38 & 45 & 66 & 24 \\
\hline Mini-Mental State Examination score, median & 24 & 14 & 13 & 8 & 16 \\
\hline ApoE e4 allele, ${ }^{\text {b }} \%$ & 24 & 29 & 28 & 28 & 29 \\
\hline History of emotional problems, \% & 20 & 13 & 11 & 11 & 17 \\
\hline Previous stroke, heart attack or diabetes, \% & 22 & 35 & 29 & 33 & 37 \\
\hline Poor self-reported health, \% & 31 & 42 & 34 & 40 & 42 \\
\hline
\end{tabular}

\begin{tabular}{|c|c|c|c|c|}
\hline \multirow[b]{2}{*}{ Symptoms } & \multirow[b]{2}{*}{ Assessment } & \multicolumn{3}{|c|}{ Population prevalence of symptoms, \% } \\
\hline & & $\begin{array}{l}\text { Participants without } \\
\text { dementia }(n=2050)\end{array}$ & $\begin{array}{l}\text { Participants with } \\
\text { dementia }(n=587)\end{array}$ & $P$ \\
\hline Apathy & $\begin{array}{l}\text { Lack of interest in things generally or in hobbies and previous interests; severe } \\
\text { listlessness; severe slowing in thinking }\end{array}$ & 12.1 & 50.3 & $<0.001$ \\
\hline Sleep problem & $\begin{array}{l}\text { Difficulty in getting to sleep and staying asleep at night, or problems with falling asleep } \\
\text { during the day }\end{array}$ & 43.8 & 42.0 & 0.574 \\
\hline Irritability & $\begin{array}{l}\text { Participant has been more angry or irritable lately, interviewer observes severe hostility } \\
\text { or angry responses }\end{array}$ & 12.8 & 28.8 & $<0.001$ \\
\hline Persecution & $\begin{array}{l}\text { Participant believes that someone is trying to upset them or harm them, or that } \\
\text { people are laughing at them. Interviewee makes false accusations or is suspicious } \\
\text { or mistrusting. Excluded if interviewer feels that beliefs are likely to be true }\end{array}$ & 8.1 & 25.4 & $<0.001$ \\
\hline Depression & $\begin{array}{l}\text { Assessed using Cambridge Mental Disorders of the Elderly Examination depression } \\
\text { algorithm }\end{array}$ & 8.6 & 20.5 & $<0.001$ \\
\hline Misidentifications & $\begin{array}{l}\text { Tendency to misidentify people or objects. Participant feels that special messages are } \\
\text { being sent on the television or radio or that their mind or body are being controlled in } \\
\text { other ways }\end{array}$ & 3.0 & 20.3 & $<0.001$ \\
\hline Hallucinations & Hearing voices; visions; smells or gustatory hallucinations & 3.7 & 15.1 & $<0.001$ \\
\hline Wandering & Getting lost or wandering has become a problem & 0.3 & 12.8 & $<0.001$ \\
\hline Elation & Uncontrollable bouts of laughter; infectious gaiety; inappropriate elated mood & 3.2 & 9.5 & $<0.001$ \\
\hline Agitation & Inappropriate verbal, vocal or motor activity; observed restlessness during interview & 3.6 & 9.0 & $<0.001$ \\
\hline Anxiety & $\begin{array}{l}\text { Suffering from fear or panic attacks, often trembles or interviewer observed severe } \\
\text { bodily features of anxiety }\end{array}$ & 6.3 & 8.9 & 0.068 \\
\hline Confabulation & Untrue responses to questions without deliberate intention to mislead & 0.3 & 5.8 & $<0.001$ \\
\hline
\end{tabular}


Apathy is the most prevalent non-cognitive symptom of dementia, present in $50.3 \%$ of those with dementia compared with $12.1 \%$ of the population without dementia.

\section{Risk factors}

Online Table DS1 shows the odds ratio of association between each BPSD and investigated risk factors after adjustment for age, gender, sensory impairment and MMSE score. The unadjusted and adjusted odds ratios were not substantially different.

There were mixed associations between BPSD and cognitive function. Wandering, apathy, persecution and misidentifications were significantly more frequent in those with low MMSE scores. Irritability was more common in those in the higher functioning groups.

Vascular risk factors were associated with a lower risk of misidentification and confabulation but were risk factors for apathy and depression, although the association with depression was not statistically significant after correction for multiple testing $(P=0.019)$. Anxiety and depression are also less likely to be found in older people with dementia, but are more common in people with worse self-reported health.

A family history of psychiatric disorders is an indicator for all BPSD except for depression, anxiety and sleep disorders, for which a personal history of emotional problems is a risk factor.

Current emotional state, measured by a recent major loss, is associated with an increased occurrence of both elation and depression, sleeping problems, apathy and hallucinations.

\section{Co-occurrence}

Online Table DS2 summarises the information about the cooccurrence of pairs of symptoms. The number of interviewees for each pair of symptoms was reported and is shown in the bottom half of the table, with the total number of interviewees reporting each symptom on the diagonal. The top half includes adjusted odds ratios for the occurrence of each symptom given the presence of each of the other symptoms.

There is a significant association between most pairs of symptoms despite adjustment for age, gender, MMSE score and sensory abilities. Anxiety and depression were very strongly associated. The symptoms of psychosis, that is misidentification, persecution and hallucination, were strongly associated with one another. Psychotic symptoms were also strongly associated with apathy.

Factor analysis of the correlation of symptoms strongly supports a solution including four factors. The rotated factor loadings are shown in Table 3. The first factor includes symptoms associated with psychosis, as well as apathy and wandering. The second factor includes anxiety and depression, and neither of these symptoms loads significantly onto any other factor. Irritability loads strongly onto a single factor and is associated with feelings of persecution and agitation. A fourth factor is comprised of elated mood, sleep disorders, hallucinations, agitation and wandering.

The uniqueness of each symptom in the factor model measures the proportion of variability of each symptom not explained by the factor model. This shows that although the factor analysis accounts for the co-occurrence of the symptoms well, a large amount of the individual variability of the symptoms cannot be explained in this way.

\section{Persistence and incidence of BPSD}

Of the 587 participants with dementia assessed at the baseline assessment, $244(42 \%)$ were reassessed 2 years later. Of the 343 participants who did not provide a second interview, 219 had died, 53 were not able to provide a second interview, 63 refused a second interview and 8 had moved away. The median time between the first and second assessment was 22 months (range 12-35, interquartile range 21-24).

Of the 12417 participants not diagnosed with dementia at baseline, 8583 were reassessed and 326 were diagnosed with dementia at the second wave of interviews. The clinical and demographic details of the prevalent and incident dementia groups at wave 2 are shown in Table 1. Our results were not significantly affected by refusal to be interviewed at follow-up.

Table 4 shows the numbers of participants with and without each symptom at baseline, the number for whom the symptom was present at both assessments and the number in whom the symptom was first seen at follow-up. The odds ratio of symptom presence at follow-up depending on the presence at baseline is also shown.

The presence of apathy, elated mood or confabulation at follow-up was not significantly linked to their presence at baseline. Conversely, anxiety, depression and wandering behaviour at baseline were strong indicators for their presence at follow-up. Symptoms of psychosis, sleeping problems, agitation and irritability were more often observed at follow-up in participants who showed them at baseline, but also occurred in participants who did not.

Disorders of mood, that is anxiety, depression and elation, did not tend to persist. We found that $18 \%$ of participants with depression at baseline had depression after 2 years, and anxiety

\begin{tabular}{|c|c|c|c|c|c|}
\hline Symptom loading & Factor 1 & Factor 2 & Factor 3 & Factor 4 & Uniqueness \\
\hline Agitation & 0.27 & 0.08 & 0.37 & 0.35 & 0.64 \\
\hline Anxiety & 0.01 & 0.76 & 0.05 & 0.11 & 0.41 \\
\hline Apathy & 0.69 & 0.20 & 0.16 & 0.09 & 0.45 \\
\hline Confabulation & 0.34 & -0.06 & -0.04 & -0.08 & 0.73 \\
\hline Depression & 0.07 & 0.79 & 0.06 & -0.04 & 0.36 \\
\hline Elation & 0.25 & 0.04 & 0.09 & 0.54 & 0.63 \\
\hline Hallucination & 0.47 & 0.42 & 0.06 & 0.34 & 0.44 \\
\hline Irritability & 0.09 & 0.11 & 0.58 & -0.01 & 0.64 \\
\hline Misidentification & 0.78 & -0.03 & 0.08 & 0.09 & 0.36 \\
\hline Persecution & 0.35 & 0.09 & 0.51 & 0.27 & 0.52 \\
\hline Sleep problems & 0.18 & 0.19 & 0.24 & 0.37 & 0.74 \\
\hline Wandering & 0.54 & -0.01 & 0.11 & 0.38 & 0.55 \\
\hline
\end{tabular}


Table 4 The profile of behavioural and psychological symptoms of dementia at baseline and follow-up

\begin{tabular}{|c|c|c|c|c|c|c|c|c|c|}
\hline & \multicolumn{7}{|c|}{ Dementia at baseline $(n=244)$} & \multirow{2}{*}{\multicolumn{2}{|c|}{$\begin{array}{l}\text { No dementia at baseline } \\
\qquad(n=348)\end{array}$}} \\
\hline & \multicolumn{3}{|c|}{ Symptom absent at baseline } & \multicolumn{3}{|c|}{ Symptom present at baseline } & \multirow[b]{2}{*}{ OR (95\% Cl) } & & \\
\hline & $n$ & $\begin{array}{l}\text { Symptom } \\
\text { present at } \\
\text { follow-up, } n\end{array}$ & $\%(95 \%$ Cl) & $n$ & $\begin{array}{l}\text { Symptom } \\
\text { present at } \\
\text { follow-up, } n\end{array}$ & $\%(95 \% \mathrm{Cl})$ & & $\begin{array}{l}\text { Symptom present } \\
\text { at first dementia } \\
\text { diagnosis, } n\end{array}$ & $\%(95 \% \mathrm{Cl})$ \\
\hline Apathy & 111 & 68 & $61(52-70)$ & 133 & 88 & $66(57-73)$ & $1.2(0.7-2.2)$ & 163 & $47(41-52)$ \\
\hline Irritability & 166 & 43 & 25 (19-32) & 78 & 40 & $51(40-62)$ & $3.0(1.7-5.5)$ & 140 & 40 (35-46) \\
\hline Wandering & 195 & 23 & $11(7-16)$ & 49 & 29 & $59(45-72)$ & $10.8(5.0-23.6)$ & 37 & 11 (8-14) \\
\hline Elation & 216 & 19 & $8(5-12)$ & 28 & 4 & $14(4-29)$ & $1.7(0.4-5.8)$ & 25 & $7 \quad(5-10)$ \\
\hline Depression & 201 & 9 & $4(2-7)$ & 43 & 8 & $18(8-31)$ & $4.9(1.5-15.2)$ & 54 & $16(12-20)$ \\
\hline Anxiety & 225 & 4 & $2(0-4)$ & 19 & 5 & $26(9-47)$ & $19.7(3.7-107.9)$ & 25 & $7(5-10)$ \\
\hline Sleep problems & 177 & 36 & $20(14-26)$ & 67 & 30 & $44(33-56)$ & $3.2(1.7-6.1)$ & 100 & $29(24-34)$ \\
\hline Agitation & 217 & 40 & $18(13-23)$ & 27 & 11 & $40(23-59)$ & $3.0(1.2-7.6)$ & 47 & $14(10-18)$ \\
\hline Misidentification & 184 & 52 & $28(22-34)$ & 60 & 30 & $50(37-62)$ & $2.5(1.3-4.8)$ & 53 & $15(12-19)$ \\
\hline Confabulation & 229 & 17 & $7(4-11)$ & 15 & 2 & $13(1-33)$ & $1.9(0.2-9.6)$ & 18 & $5 \quad(3-8)$ \\
\hline Persecution & 173 & 33 & $19(13-25)$ & 71 & 30 & $42(31-53)$ & $3.1(1.6-5.9)$ & 98 & $28(23-33)$ \\
\hline Hallucinations & 198 & 26 & $13(8-18)$ & 46 & 16 & $34(21-48)$ & $3.5(1.6-7.8)$ & 51 & $15(11-19)$ \\
\hline
\end{tabular}

was seen in $26 \%$ of participants with anxiety at baseline. The incidence of both symptoms was also very low, with depression present at follow-up in $4 \%$ of those without depression at baseline, and anxiety only present at follow-up in $2 \%$ of those without anxiety at baseline. Elated mood was present in 4 (14\%) of the 28 participants in whom it was seen at baseline and was seen in $19(8 \%)$ of the 216 in whom it was not.

Symptoms of psychosis were more persistent. Persecutory delusions were persistent in 30 of the 71 cases $(42 \%)$ and misidentification was persistent in 30 out of $60(50 \%)$. We also found a high incidence of misidentifications, with $28 \%$ of participants who did not misidentify people or objects at baseline doing so by the follow-up interview. Hallucinations had lower incidence and persistence than other symptoms of psychosis.

The temporal association between each of the factors described in Table 3 is shown in Table 5. There is an association between the level for each of the factor scores at baseline and the same factor at follow-up. Despite the independence of the factor scores at baseline, there are statistically significant associations between levels of factor 1 (psychosis, apathy) at baseline and factor 4 (sleep disorders, hallucinations and wandering) at follow-up, and between factor 4 at baseline and factor 1 at follow-up.

The prevalence of symptoms in the 348 participants developing dementia between the first and second waves of interviews is also shown in Table 4. The profile of symptoms in this group is similar to that seen in the general population with dementia (Table 2). Only irritability is significantly more common in this incident dementia group than in the prevalent population with dementia.

\section{Discussion}

\section{Summary of results}

We have estimated the prevalence, co-occurrence, risk factors and course of BPSD using a large cohort representative of the population over 65 in England and Wales. This was compared with the prevalence of the same symptoms in the population without dementia. All of the behavioural and psychological symptoms we investigated except for sleep disorders were found to be significantly more common in the population with dementia, although disorders of mood, apathy, irritability and feelings of persecution were also seen in a significant proportion of the population without dementia. These symptoms in individuals with dementia should, therefore, not necessarily be assumed to be a consequence of their dementia. Other symptoms including psychosis, wandering and confabulation were substantially more common in people with dementia and their presence increased with increasing impairment.

There are associations between many pairs of symptoms and these can be described by a factor model including four factors. These factors corresponded to psychosis and apathy, anxiety and depression, irritability and persecution and a fourth factor comprised of elated mood, sleep disorders, hallucinations, agitation and wandering. The high uniqueness of each symptom shows that the factor model does not provide a complete description of the pattern of symptom occurrence and that symptoms must still be considered individually.

The presence of symptoms is affected by a combination of clinical and demographic risk factors as well as the circumstances of the individual. Most of the symptoms we examined were more likely to be present at a 2-year follow-up in the participants in whom they were seen at baseline, although the strength of this association varied across symptoms.

\section{Strengths and weaknesses of the study}

The main strengths of this study of the epidemiology of BPSD are the population setting of the study and its size. Our assessment used interviews with both participants and their caregivers, and enabled a wide range of demographic and clinical factors to be investigated. A limitation of our assessment is that only the presence or absence of symptoms and neither their severity nor the extent to which they were problematic could be recorded. The GMS examination is not designed specifically for the assessment of BPSD. Our definition of misidentification aimed to capture any delusional ideation excluding persecutory

\begin{tabular}{|c|c|c|c|c|}
\hline \multirow[b]{2}{*}{ Baseline factor score } & \multicolumn{4}{|c|}{ Follow-up factor score } \\
\hline & Factor 1 & Factor 2 & Factor 3 & Factor 4 \\
\hline Factor 1 & 0.27 & 0.05 & -0.03 & 0.23 \\
\hline Factor 2 & -0.07 & 0.36 & 0.09 & 0.04 \\
\hline Factor 3 & 0.04 & -0.02 & 0.28 & 0.08 \\
\hline Factor 4 & 0.20 & 0.02 & 0.10 & 0.20 \\
\hline
\end{tabular}


delusions, but the tendency to misidentify objects can also be considered a direct symptom of cognitive impairment and this symptom was very rare in participants without dementia and those with high MMSE scores.

We did not adjust for any medication or other interventions aimed at reducing BPSD. Reported use of antipsychotic medications in people with dementia was rare, rising from $3 \%$ at baseline to $6 \%$ at year ten. Cholinesterase inhibitors were being taken by fewer than $5 \%$ of respondents with dementia at the 10-year follow-up. ${ }^{17}$

Findings regarding the association between BPSD and risk factors were adjusted for age, gender, MMSE score and any visual or hearing impairment that might contribute to or prevent the assessment of symptoms. Our results did not change substantially following these adjustments. Owing to the large number of statistical tests included in our analyses we controlled the false discovery rate at $5 \%$, but it remains possible that there are some false associations.

Between the baseline and 2-year assessments, 58\% of participants with dementia died or were otherwise lost to follow-up. This level of attrition over 2 years is similar to that in comparable studies. Our baseline and follow-up interviews were 2 years apart and so we were not able to observe any short-term fluctuations or short episodes of symptoms.

\section{Comparisons with previous studies}

Several previous longitudinal studies of BPSD have been conducted with the aim of describing the occurrence and progression of the different symptoms throughout the course of dementia. These studies have taken place in a variety of settings, including out-patient clinics ${ }^{18,19}$ residential homes ${ }^{20,21}$ or in samples drawn from the population. ${ }^{3,4,22,23}$ The findings between studies have not always been consistent, although many of the differences are attributable to their settings, design, instruments and sample size, and despite these differences, a consistent picture of the epidemiology of BPSD is emerging.

The prevalence of BPSD in the population has been estimated from the Nakayama study, ${ }^{4}$ the Cache County Study $(n=201)^{24}$ and the Cardiovascular Health Study. ${ }^{3}$ Each of these studies used the Neuropsychiatric Inventory (NPI) and their results are largely consistent. Our results match their findings, except that our estimates for the prevalence of anxiety (8.9\%) and agitation $(9.0 \%)$ are significantly lower and the prevalence of apathy $(50.3 \%)$ is higher. These differences could be explained by the difference in instruments used to measure the symptoms. For example, in addition to the type of questions asked in the NPI to measure apathy, our assessment also includes a self-report of listlessness. We further found that apathy at baseline does not affect the presence of apathy at follow-up, which indicates that all people with dementia are at risk of developing the symptom. In the 244 participants who underwent both baseline and 2-year assessments, only $43(18 \%)$ were not diagnosed with apathy at either assessment.

The extent to which BPSD are simply manifestations of cognitive impairment remains unclear. It has been reported that BPSD are rarely observed in the terminal stages of dementia, ${ }^{25}$ although as stated in a previous review 'cognitive function directly contributes to the emergence and severity of BPSD, wandering explained as an expression of loneliness or hunger, agitated behaviours resulting from the natural frustration of dyspraxia or forgetfulness, and misidentifications developing from pathological damage to the visual cortex. ${ }^{25}$ The Cache County Study reported that a greater dementia severity was associated with increased presence of all BPSD included on the ten-item NPI except for depression, for which a non-significant negative association was observed. $^{5}$

We found psychosis to be more common in institutionalised participants and those with drinking problems or a family history of psychiatric disorder. Psychotic symptoms were relatively persistent and psychosis was most often seen in those with moderate or severe cognitive impairment. In a review of 55 studies published between 1990 and 2003 on the epidemiology of and risk factors for psychosis in Alzheimer's disease, ${ }^{26}$ more severe cognitive impairment was also associated with higher rates of psychosis, whereas gender, education and family history of dementia or psychiatric illness showed a weak or inconsistent relationship with psychosis. Although no other longitudinal study has considered persecutory delusions separately from misidentifications or other delusional ideation, delusional ideation has previously been reported to be persistent over long intervals. ${ }^{23}$

The co-occurrence between symptoms of depression and anxiety is frequently reported, as well as a low association between these and other BPSD. Longitudinal studies of depression in dementia in a care home setting ${ }^{21}$ and in psychiatric out-patient units $^{17,19,27}$ have discovered low persistence and low incidence. Our study confirms in a large population-based cohort what has been previously observed in these smaller clinical and care home settings. The Cache County Study reported higher persistence ${ }^{23}$ and incidence ${ }^{22}$ of mood disorders. The most likely explanation for this difference is that the group of participants considered to be at risk of developing each symptom were those with no symptoms at all at baseline. This group were less cognitively impaired, with a mean MMSE score of 18 , than were our baseline sample.

The prevalence of depression and anxiety in the incident dementia group is similar to that seen in the baseline cohort. This finding, combined with their low incidence in those with dementia supports the idea that depression and anxiety arise around the time of onset of dementia and are less likely to occur in later stages of the disease. Depression was related to a history of heart attack or stroke, which although not meeting our threshold for statistical significance supports the previous finding that these symptoms are more common in vascular dementia ${ }^{27}$ and with the hypothesis of a vascular contribution to depression in the older population. ${ }^{28}$ In the Cache County Study, depression was less commonly seen in people with Alzheimer's disease ${ }^{5}$ than in all dementia, but no difference was seen in rates of psychosis. ${ }^{8} \mathrm{~A}$ further analysis of vascular risk for BPSD in individuals with Alzheimer's disease from the Cache County Study found a higher rate of depression, apathy and delusions in those with a history of stroke, and more agitation, anxiety and delusions in those with hypertension. $^{29}$

In common with other studies, we have found elated mood to be a rare symptom in dementia and as such its distribution in the population is difficult to measure precisely. Our results and those from previous studies ${ }^{18,22,23}$ suggest that where they do occur, episodes of elated mood in dementia do not persist.

Sleeping problems as defined by current neuropsychiatric scales are common in all stages of dementia as well as in the elderly population without dementia. A more detailed assessment than those currently used is needed to measure the pattern of dementia-related sleeping problems in the population.

Wandering and agitation were more common in those with an ApoE e4 allele and although the associations were not statistically significant after our false discovery rate correction, they are consistent with previous findings. Aberrant motor behaviour, defined in a similar way to our definition of agitation, was the only NPI item found to be associated with ApoE genotype in the Cache County Study. ${ }^{5}$ This suggests that these symptoms are 
a consequence of neuropathology related to Alzheimer's disease. Previous studies of associations between ApoE genotype and BPSD within Alzheimer's disease cohorts have been inconclusive. ${ }^{30}$

Our factor structure for the co-occurrence of symptoms agrees to some extent with that discovered by previous clinical studies $^{31-33}$ which consistently report a factor including psychotic symptoms, a factor involving depression and anxiety and a factor including irritability and agitation. A recent study of Dutch nursing home residents found this structure to vary little across dementia stages. ${ }^{34}$ An alternative approach to describe the co-occurrence of symptoms is cluster analysis, where patients are grouped based on their symptom profile. This leads to nonoverlapping clusters of patients, with the symptom profile of each patient assumed to be solely dependent on their class. ${ }^{24,32,35}$ These analyses typically result in a class structure that agrees with our factor structure. However, we have shown that the levels of psychotic and affective symptoms are largely independent, and so any classification that forces people into one or other of these categories makes those exhibiting both classes of symptoms impossible to classify.

The course of BPSD has previously been compared across dementia subtypes. Visual hallucinations have been shown to be more common and more persistent in dementia with Lewy bodies than in individuals with Alzheimer's disease. ${ }^{36,37}$ People with vascular dementia are thought to have more persistent depression. ${ }^{38}$ We have not reported symptoms by dementia subtype, partly because brain findings in this population sample have revealed a high proportion of mixed pathology. ${ }^{39}$

\section{Clinical implications}

These results have implications for clinical practice, in the development and assessment of treatments and in understanding the aetiology of BPSD. They will assist prediction of the future symptom profile of an individual with dementia given their current profile, and the symptoms that are expected to occur around the time of dementia onset.

Many of the behavioural and psychological symptoms associated with dementia are also present in the older population without dementia and so not every non-cognitive symptom observed in a person with dementia can be assumed to be a symptom of their dementia. There are many clinical and socio-demographic factors that are associated with the presence of BPSD. These will enable the development of interventions to better manage symptoms and to predict their occurrence.

Our results support previous attempts to describe the co-occurrence of symptoms, but we also observed a high level of symptom uniqueness, that is, the factor solution we described could not account for much of the symptom variability. Therefore, although relationships between individual symptoms undoubtedly exist, a syndrome approach to their study and management or attempts to classify individuals based on their profile of symptom occurrence might not be appropriate.

\footnotetext{
George M. Savva, PhD, Julia Zaccai, PhD, Department of Public Health and Primary Care, University of Cambridge; Fiona E. Matthews, PhD, MRC Biostatistics Unit Institute of Public Health, Cambridge; Julie E. Davidson, MPH, Worldwide Epidemiology, GlaxoSmithKline R\&D, Harlow; Ian McKeith, MD, FRCPsych, FMedSci Epidemiology, GlaxoSmithKline R\&D, Harlow; lan McKeith, MD, FRCPsych, FMedSCi,
Wolfson Research Centre, Newcastle General Hospital, Newcastle Upon Tyne; Carol Brayne, MD, MSC, FRCP, FFPH, Department of Public Health and Primary Care, University of Cambridge, UK.

Correspondence: George M. Savva, Department of Public Health and Primary Care, University of Cambridge, Institute of Public Health, University Forvie Site, Robinson Way, Cambridge CB2 OSR, UK. Email: george.savva@phpc.cam.ac.uk

First received 11 Jan 2008, final revision 9 Jun 2008, accepted 27 Aug 2008
}

\section{References}

1 Tariot PN, Mack JL, Patterson MB, Edland SD, Weiner MF, Fillenbaum G, et al. The Behavior Rating Scale for Dementia of the Consortium to Establish a Registry for Alzheimer's Disease. The Behavioral Pathology Committee of the Consortium to Establish a Registry for Alzheimer's Disease. Am J Psychiatry 1995; 152: 1349-57.

2 Shin IS, Carter M, Masterman D, Fairbanks L, Cummings JL. Neuropsychiatric symptoms and quality of life in Alzheimer disease. Am J Geriatr Psychiatry 2005; 13: 469-74.

3 Lyketsos CG, Lopez O, Jones B, Fitzpatrick AL, Breitner J, DeKosky S. Prevalence of neuropsychiatric symptoms in dementia and mild cognitive impairment: results from the cardiovascular health study. JAMA 2002; 288: 1475-83.

4 Ikeda M, Fukuhara R, Shigenobu K, Hokoishi K, Maki N, Nebu A, et al. Dementia associated mental and behavioural disturbances in elderly people in the community: findings from the first Nakayama study. $J$ Neurol Neurosurg Psychiatry 2004; 75: 146-8.

5 Steinberg M, Corcoran C, Tschanz JT, Huber C, Welsh-Bohmer K, Norton MC, et al. Risk factors for neuropsychiatric symptoms in dementia: the Cache County Study. Int J Geriatr Psychiatry 2006; 21: 824-30.

6 Harwood DG, Barker WW, Ownby RL, Duara R. Relationship of behavioral and psychological symptoms to cognitive impairment and functional status in Alzheimer's disease. Int J Geriatr Psychiatry 2000; 15: 393-400.

7 Hope T, Keene J, McShane RH, Fairburn CG, Gedling K, Jacoby R. Wandering in dementia: a longitudinal study. Int Psychogeriatr 2001; 13: 137-47.

8 Leroi I, Voulgari A, Breitner JC, Lyketsos CG. The epidemiology of psychosis in dementia. Am J Geriatr Psychiatry 2003; 11: 83-91.

9 Starkstein SE, Jorge R, Mizrahi R, Robinson RG. A prospective longitudinal study of apathy in Alzheimer's disease. J Neurol Neurosurg Psychiatry 2006; 77: 8-11.

10 Brayne C, Mccracken C, Matthews FE. Cohort profile: the Medical Research Council Cognitive Function and Ageing Study (CFAS). Int J Epidemiol 2006; 35: $1140-5$

11 Folstein MF, Folstein SE, McHugh PR. 'Mini-mental state'. A practical method for grading the cognitive state of patients for the clinician. J Psychiatr Res 1975; 12: 189-98.

12 Copeland JR, Dewey ME, Griffiths-Jones HM. A computerized psychiatric diagnostic system and case nomenclature for elderly subjects: GMS and AGECAT. Psychol Med 1986; 16: 89-99.

13 Copeland JR, Dewey ME, Griffiths-Jones HM. A computerized psychiatric diagnostic system and case nomenclature for elderly subjects: GMS and AGECAT. Psychol Med 1986; 16: 89-99.

14 Roth M, Tym E, Mountjoy CQ, Huppert FA, Hendrie H, Verma S, et al. CAMDEX. A standardised instrument for the diagnosis of mental disorder in the elderly with special reference to the early detection of dementia. $\mathrm{Br} J$ Psychiatry 1986; 149: 698-709.

15 Benjamini $Y$, Hochberg $Y$. Controlling the false discovery rate - a practical and powerful approach to multiple testing. J R Stat Soc Series $B$ Methological 1995; 57: 289-300.

16 Eustace A, Coen R, Walsh C, Cunningham CJ, Walsh JB, Coakley D, et al A longitudinal evaluation of behavioural and psychological symptoms of probable Alzheimer's disease. Int J Geriatr Psychiatry 2002; 17: 968-73.

17 Matthews FE, McKeith I, Bond J, Brayne C, MRC CFAS. Reaching the population with dementia drugs: what are the challenges? Int J Geriatr Psychiatry 2006; 22: 627-31.

18 Aalten $\mathrm{P}$, de Vugt ME, Jaspers $\mathrm{N}$, Jolles J, Verhey FR. The course of neuropsychiatric symptoms in dementia. Part I: findings from the two-yea longitudinal Maasbed study. Int J Geriatr Psychiatry 2005; 20: 523-30.

19 Ryu SH, Katona C, Rive B, Livingston G. Persistence of and changes in neuropsychiatric symptoms in Alzheimer disease over 6 months: the LASERAD study. Am J Geriatr Psychiatry 2005; 13: 976-83.

20 Ballard CG, Margallo-Lana M, Fossey J, Reichelt K, Myint P, Potkins D, et al. A 1-year follow-up study of behavioral and psychological symptoms in dementia among people in care environments. J Clin Psychiatry 2001; 62: 631-6.

21 Payne JL, Sheppard JM, Steinberg M, Warren A, Baker A, Steele C, et al. Incidence, prevalence, and outcomes of depression in residents of a longterm care facility with dementia. Int J Geriatr Psychiatry 2002; 17: 247-53.

22 Steinberg M, Sheppard JM, Tschanz JT, Norton MC, Steffens DC, Breitner JC, et al.The incidence of mental and behavioral disturbances in dementia: the cache county study. J Neuropsychiatry Clin Neurosci 2003; 15: 340-5.

23 Steinberg M, Tschanz JT, Corcoran C, Steffens DC, Norton MC, Lyketsos CG, et al. The persistence of neuropsychiatric symptoms in dementia: the Cache County Study. Int J Geriatr Psychiatry 2004; 19: 19-26. 
24 Lyketsos CG, Sheppard JM, Steinberg M, Tschanz JA, Norton MC, Steffens DC et al. Neuropsychiatric disturbance in Alzheimer's disease clusters into three groups: the Cache County study. Int J Geriatr Psychiatry 2001; 16: 1043-53.

25 Mcllroy S, Craig D. Neurobiology and genetics of behavioural syndromes of Alzheimer's disease. Curr Alzheimer Res 2004; 1: 135-42.

26 Ropacki SA, Jeste DV. Epidemiology of and risk factors for psychosis of Alzheimer's disease: a review of 55 studies published from 1990 to 2003. Am J Psychiatry 2005; 162: 2022-30.

27 Ballard C, Bannister C, Solis M, Oyebode F, Wilcock G. The prevalence, associations and symptoms of depression amongst dementia sufferers. J Affect Disord 1996; 36: 135-44.

28 Alexopoulos GS, Meyers BS, Young RC, Campbell S, Silbersweig D, Charlson M. 'Vascular depression' hypothesis. Arch Gen Psychiatry 1997; 54: 915-22.

29 Treiber KA, Lyketsos CG, Corcoran C, Steinberg M, Norton M, Green RC, et al. Vascular factors and risk for neuropsychiatric symptoms in Alzheimer's disease: the Cache County Study. Int Psychogeriatr 2008; 20: 538-53.

30 Pritchard AL, Harris J, Pritchard CW, Coates J, Haque S, Holder R, et al. The effect of the apolipoprotein $\mathrm{E}$ gene polymorphisms and haplotypes on behavioural and psychological symptoms in probable Alzheimer's disease. J Neurol Neurosurg Psychiatry 2007; 78: 123-6.

31 Aalten $P$, de Vugt ME, Lousberg R, Korten E, Jaspers N, Senden B, et al. Behavioral problems in dementia: a factor analysis of the neuropsychiatric inventory. Dement Geriatr Cogn Disord 2003; 15: 99-105.

32 Aarsland D, Bronnick K, Ehrt U, De Deyn PP, Tekin S, Emre M, et al. Neuropsychiatric symptoms in patients with Parkinson's disease and dementia: frequency, profile and associated care giver stress. J Neurol Neurosurg Psychiatry 2007; 78: 36-42.
33 Amer-Ferrer G, de la Pena A, Garcia Soriano MT, Garcia Martin A. Main components of Neuropsychiatric Inventory in Alzheimer's disease. Definition of behavioral syndromes [Spanish]. Neurologia 2005; 20: 9-16.

34 Zuidema SU, de Jonghe JF, Verhey FR, Koopmans RT. Neuropsychiatric symptoms in nursing home patients: factor structure invariance of the Dutch nursing home version of the neuropsychiatric inventory in different stages of dementia. Dement Geriatr Cogn Disord 2007; 24: 169-76.

35 Moran M, Walsh C, Lynch A, Coen RF, Coakley D, Lawlor BA. Syndromes of behavioural and psychological symptoms in mild Alzheimer's disease. Int $J$ Geriatr Psychiatry 2004; 19: 359-64.

36 Ballard CG, O'Brien JT, Swann AG, Thompson P, Neill D, McKeith IG. The natural history of psychosis and depression in dementia with Lewy bodies and Alzheimer's disease: persistence and new cases over 1 year of followup. J Clin Psychiatry 2001; 62: 46-9.

37 Stavitsky K, Brickman AM, Scarmeas N, Torgan RL, Tang MX, Albert M, et al. The progression of cognition, psychiatric symptoms, and functional abilities in dementia with Lewy bodies and Alzheimer disease. Arch Neurol 2006; 63 1450-6.

38 Starkstein SE, Mizrahi R, Garau L. Specificity of symptoms of depression in Alzheimer disease: a longitudinal analysis. Am J Geriatr Psychiatry 2005; 13 802-7.

39 Pathological correlates of late-onset dementia in a multicentre, communitybased population in England and Wales. Neuropathology Group of the Medical Research Council Cognitive Function and Ageing Study (MRC CFAS). Lancet 2001; 357: 169-75.

\section{Why borderline baulks mainstream psychiatry}

Medical and psychiatric treatments contain three indispensable components: a therapeutic relationship, meaning-making and changepromotion. For people who have borderline personality disorder each is problematic. Relationships are chaotically sought or fled from; meaning equates to control or irrelevancy; naive attempts at change invalidate precarious defensive 'solutions' to despair or overwhelming affect, such as self-harm or addiction. Conventional approaches thus typically exacerbate rather than alleviate distress. Effective treatments for borderline personality disorder: tolerate and target disruptions to the therapeutic relationship; start from the client's own meaning structures before co-constructing new ones; and validate while simultaneously introducing changes in thought patterns and behaviour. 